Marieflor Cristy M. Garcia, MD Charlotte M. Chiong, MD ${ }^{1,2}$ Generoso T. Abes, MD, MPH ${ }^{1,2}$

Ryner Jose C. Carrillo, MD, MSC'

'Department of Otorhinolaryngology

Philippine General Hospital

University of the Philippines Manila

2Philippine National Ear Institute University of the Philippines, Manila

National Institutes of Health

\section{Accuracy of Reflexive Behavioral ("Baah") Test in the Screening for Hearing Impairment in Infants Six Months Old and Below}

\begin{abstract}
Objective: To determine the prevalence of hearing loss among infants six months old and below sent for newborn hearing screening in our institution, and to measure the accuracy, sensitivity, specificity and positive predictive values of reflexive behavioral ("Baah") test in detecting hearing loss in infants.
\end{abstract}

\section{Methods:}

Design: Cross-sectional study

Setting: Ear Unit of a tertiary government hospital

Participants: Infants less than Six months old sent for newborn hearing screening at the Ear Unit of a tertiary government hospital from April to September, 2011 were recruited. All participants were tested with OAE for hearing screening. OAE was also used as the standard for evaluating hearing impairment. The reflexive behavioral ("Baah") test was then done using the human voice as a loud sound stimulus, and the response recorded were auropalpebral, startle and blinking response to the sound. The sensitivity, specificity, accuracy, positive and negative predictive value of the test was then measured.

Results: From April to September 2011, a total of 101 patients were tested, with a male to female ratio of 1.1:1 (53 males, 48 females). The prevalence of hearing impairment in this study population was $6.9 \%$ (7 out of 101). The reflexive behavioral ("Baah") test was found to have sensitivity of $71.4 \%$, specificity of $95.7 \%$, accuracy rate of $94 \%$, positive predictive value of $55.6 \%$ and negative predictive value of $97.8 \%$.

Conclusion: The reflexive behavioral ("Baah") test shows potential as an accurate, acceptable and cost-effective screening tool to identify infants that may be at higher risk for hearing impairment. This test may aid the health care providers, in areas without OAEs, in identifying infants who are in need further hearing diagnostic evaluation, with OAEs or other hearing tests. It is recommended that the "Baah" test be implemented in the community to test its reproducibility in a larger population and outside the hospital setting.

Keywords: reflexive behavioral test, "Baah" test, otoacoustic emission, hearing screening 


\section{ORIGINAL ARTICLE}

Language impairment due to hearing loss is a preventable condition, provided that it is diagnosed and managed early in life. Hearing loss, if not detected and addressed at an early age, not only leads to speech and language difficulties, but may also lead to poor cognitive, social, and emotional development. ${ }^{1}$ This reality has led several countries and organizations to advocate a universal system of newborn hearing screening in order to ensure early detection and provide opportunities for early intervention. In the United States, several groups such as the Joint Committee on Infant Hearing, the American Academy of Pediatrics, Centers for Disease Control and Prevention, and Healthy People 2010 have formulated and implemented recommendations. By 2007, 37 states in America had enacted legislation requiring that hearing screening be performed on all newborns in hospitals and birthing centers. ${ }^{2}$

At present, the accepted standard of care for newborn hearing screening is Otoacoustic Emissions (OAE) and/or Auditory Brainstem Response (ABR). These are reliable tests that are based on physiologic responses, making the results objective, in contrast to other tests that rely on an observer's assessment of specific responses. Different countries and hospitals have their own protocols for newborn hearing screening, such as: (1) OAEs before a newborn is discharged, followed by an outpatient OAE re-screen for those who did not pass the first $O A E s$, (2) OAE inpatient screening and ABR re-screen for those who did not pass, (3) OAE and ABR inpatient screens, and (4) ABR inpatient and $A B R$ re-screens. ${ }^{1,3,4}$ These protocols depend on the standards set by the institution, balanced by the availability of resources, so that the protocol can be applied to the most number of individuals.

In the Philippines, the newborn hearing screening act or Republic Act 9709 has already been signed into law. Its purpose is to "institutionalize measures for the prevention and early diagnosis of congenital hearing loss among newborns and the provision of referral, early intervention, counseling and other support services of newborns with hearing loss." ${ }^{15}$ However, a primary barrier to the implementation of a universal health system in developing countries such as ours is the lack of affordable, reliable, and easy-to-use methodology that can be employed in farflung communities. In the Philippines, OAE machines are usually found only in tertiary institutions or in the city, and are almost unheard of in rural areas. In a developing country, only very few local government units and hospitals are willing to invest in these instruments, as they give priority to more life-threatening and emergent health problems. ${ }^{6}$ The number of those who can operate these instruments is even more limited.

Provision of these hearing screening instruments to all the local government units in the country may take a long while given the country's present economic situation. As time passes, more and more deaf babies will grow up past the critical age with their condition undetected. The incidence of bilateral congenital profound hearing impairment is about 1.3 per 1000 Filipino newborns. ${ }^{7}$ For the year 2010, the number of live births in the Philippines was estimated to be over 2 million. ${ }^{8,9}$ With these figures, about 2,600 profoundly deaf babies are born every year, majority of which are born at home or in centers without newborn hearing screening equipment. This number still excludes those with milder forms of hearing loss that are also at risk for some degree of speech and/or language impairment.

With the desire to improve early detection of hearing loss and reach the greatest number of population, we were in search of a reliable and simple hearing screening technique.

In 1930, a study by Bryan noted that blinking, startling, and stirring reactions in response to a loud sound is typical for newborn infants. ${ }^{10}$ Several behavioral tests have then been developed relying on observation of the behavior of the infants and children being tested. Although the older literature may find some responses to be less reliable to diagnose hearing loss, the auropalpebral reflex in the newborn has been established. Similar to the moro test as an indicator for neuromuscular development of infants, the auropalpebral reflex might be useful as a screening tool for possible hearing impairment. ${ }^{10,}$ 11

In the Philippines, a pilot study was done by Abes et al., to test the validity of human voice as the loud sound stimulus that may be used to provoke reflexive reactions from infants. Results of the study showed that the sound "Baah" covers both high and low frequencies (150$5000 \mathrm{hZ}$ ) which is similar to the frequencies tested by the OAEs. This is in contrast with the other sound investigated (i.e., "Psst") that carries only high frequency tones, which, if used for hearing screening, may falsely label as profoundly hearing-impaired those with residual hearing at low frequencies. It has also been found that at a distance of 1 meter, $85 \%$ of males and females recited the word "Baah" at an intensity of $80 \mathrm{db}$ SPL or louder, in contrast with "Psst" that was produced in lower and more variable intensities. Hence, if the goal is to prioritize early detection and referral of the profoundly hearing impaired children, the sound "Baah" appears to be the more appropriate sound stimulus. ${ }^{12}$

Based on these data, this study was developed to test whether the sound "Baah" and the reflexive response of newborns may be used as a screening tool for identifying possible hearing impairment in a newborn.

The objective of the study was to determine the prevalence of hearing 
loss among infants 6 months old and below sent for newborn hearing screening in our institution, and to calculate the accuracy, sensitivity, specificity and positive predictive values of reflexive behavioral ("Baah") test in detecting hearing loss in infants.

\section{METHODS}

\section{Study Design}

This was a cross sectional study. The research protocol was presented to and approved by the Research Committee of the Department of Otorhinolaryngology of the Philippine General Hospital, and abided by the Helsinki Declaration.

\section{Setting}

The study was performed in a quiet, enclosed, air-conditioned room designated for OAE testing at the Ear Unit of the Philippine General Hospital, a tertiary national university hospital. The baby was laid on a flat bed while the parent or guardian was seated on a designated area at bedside.

\section{Participants}

From April to September 2011, parents of infants six months old or younger sent for OAE testing during the duty schedules of the tester and observer were asked to enroll their child in the study, hence, convenience sampling was used. For each of the parent or guardian, detailed information regarding the study was provided in a written form, which was supplemented by verbal explanation for all the recruits. A consent form was signed by the parent or guardian upon agreement to participate in the study.

Inclusion criteria were: (1) male and female infants from 0-6 months old, (2) with parent or guardian's informed consent, (3) with developed ear canal for OAE testing. Excluded were those (1) without consent from the parent or guardian, (2) those with ear canal deformity in which the OAE probe could not be inserted. All infants sent to the ear unit were stable and comfortable in room air since OAE testing is an elective procedure.

\section{Calibration of stimulus intensity}

Ten infants were recruited for a pilot study which were also obtained from the OAE referrals at the Ear Unit. A single designated tester was trained to provide the voice stimulus ("Baah"). These initial 10 infants were not counted in the total number of subjects in the study. The trained tester and observer were both technicians working at the Ear Unit. An A-and-C-weighted sound level meter (TES-1350A digital sound level meter, TES Electrical Electronic Corp., Taiwan) was placed beside the infant's head during the "Baah" test for all the infants. The stimulus was produced by the trained tester, whose mouth was at a set distance of one foot away from the head of the infant. The tester took two deep breaths and then produced the sound "Baah" in a sudden manner, as described by Abes, et al. ${ }^{12}$ Based on the sound level meter, the stimulus produced was consistently recorded between 80 to $95 \mathrm{~dB}$ SPL intensity during the pilot study. A single observer was trained to observe and record the response of the infants. The most common reflex demonstrated was either blinking or a more forceful and sudden shutting of the infants'already closed eyelids. Additional responses were startling and stirring reflexes demonstrated by sudden head and body movement right after the sound was produced. The observer records the response as "present" if the infant demonstrated one or more of the responses above, and "absent" if none of the above responses was observed.

\section{Intervention or observation procedures}

The baby was placed at the designated quiet room and laid on a bed. Once the baby had settled and was in a quiet state, OAE (DP-OAE) was administered in each ear using OtoRead ${ }^{\mathrm{TM}}$ OAE Test Instrument (Interacoustics ${ }^{\circledR}$, Denmark). The OAE was tested by one of the five trained audiologists designated for the day. The OAE test instrument automatically started the test once the ambient noise was acceptably low and a seal was produced with a probe in the infant's ear canal. When a "refer" result appeared, the ear probe was removed, the baby's ear was massaged to release ear canal retraction, and the test was again repeated when the infant was settled. If "refer" was again obtained, the result was recorded as "refer" in that ear. If "pass" result was obtained on the first or second test, then the result was recorded as "pass." The audiologist recorded the result on the official form and left the room.

The trained tester and observer then entered the room, blinded to the OAE result. The tester positioned herself at the vertex part of the head of the infant, with her mouth one foot away from the infant's head. The trained observer positioned herself at the side of the infant to focus on its facial reactions.

The tester then provided the stimulus "Baah" and the observer recorded the presence of absence of response. The stimulus was provided two to three times, depending on the infant's response.

The investigator then collected and tabulated the data provided in the OAE result form, as well as the data gathered by the "Baah" investigator.

\section{Main and secondary outcome measures}

The main outcome measures were presence or absence of a response reported in the OAE and presence or absence of a response in the "Baah" test.

If the OAEs were absent in either ear (a"REFER" result), it was recorded as "disease positive." The "Baah" test was recorded "test positive" if the 


\section{ORIGINAL ARTICLE}

infant failed to demonstrate any reflexive response to at least two out of three stimuli. The "Baah" test was recorded "test negative" if the infant was able to demonstrate reflexive response to at least two stimuli.

\section{Data analysis}

Data was tabulated in a $2 \times 2$ table comparing the test stimulus ("Baah") and the standard (OAE). (Table 1) Accuracy, sensitivity, specificity, positive and negative predictive values, likelihood ratios, and post-test probabilities were calculated using the Centre for Evidence-Based Medicine (CEBM) Stats Calculator. ${ }^{13}$ The $2 \times 2$ table was analyzed using the Fisher exact test, with a $P$ value of $<0.05$ considered as significant. The P value was calculated using the GraphPad Software (GraphPad Software, Inc., USA). ${ }^{14}$

The age in days and the gender of the infants were recorded, as well as the incidence of hearing impairment based on OAE.

\section{RESULTS}

From April to September 2011, a total of 101 patients, with a male to female ratio of 1.1:1 (53 males, 48 females), were tested. The mean age was 10.33 days (range 0 to 182 days). The age distribution is shown in Figure 1.

Test results for the 101 patients are shown in Table 1.The sensitivity of the "Baah" test was calculated at $71.4 \%$ with a specificity of $95.7 \%$. The positive predictive value was $55.6 \%$ and the negative predictive value was $97.8 \%$. The $P$ value was $<0.0001$, indicating that the association between the "Baah" test and OAE is statistically significant.

The likelihood ratios for positive and negative test results were 16.786 and 0.298 , respectively. (Table 2)

Seven out of 101 infants tested positive (i.e. with hearing impairment) with OAEs with a prevalence of $6.9 \%$ in this study population. The posttest probability for a positive "Baah" test was $55.6 \%$ and the post-test probability for a negative "Baah" test was $2.1 \%$.

In summary, correct (true positive and true negative) diagnosis was achieved in 95 out of 101 infants giving the test an accuracy of $94 \%$.

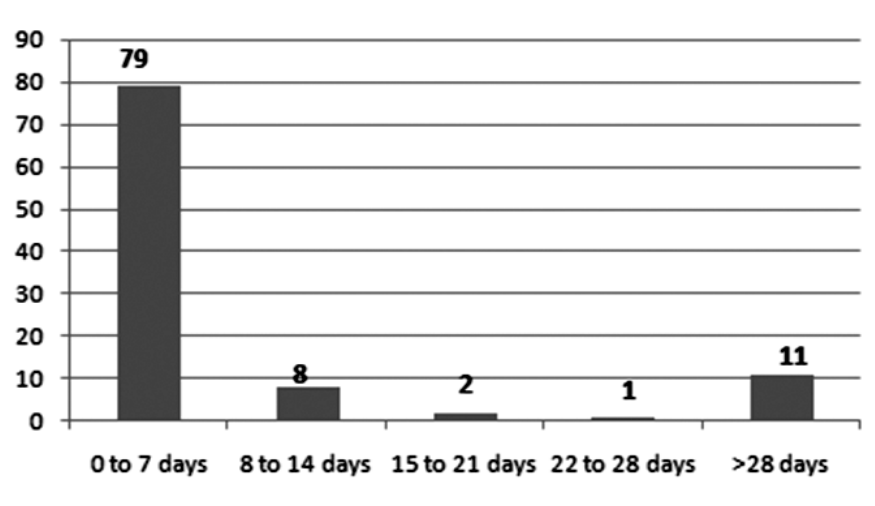

Figure 1. Age distribution of the sample population

Table 2. Likelihood ratios and post-test probabilitiesfor "Baah" test

\begin{tabular}{|c|c|}
\hline Likelihood ratio for a positive test (LR+) & $\begin{array}{l}=(\text { sensitivity }) /(1 \text {-specificity }) \\
=0.71429 /(1-0.95745) \\
=16.786\end{array}$ \\
\hline Likelihood ratio for a negative test (LR-) & $\begin{array}{l}=(1-\text { sensitivity }) / \text { specificity } \\
=(1-0.71429) / 0.95745 \\
=\mathbf{0} .298407\end{array}$ \\
\hline $\begin{array}{l}\text { Posttest Probability for a positive test } \\
\text { (PosttestProb+) }\end{array}$ & $\begin{array}{l}=\text { PretestOdds } \times L R+) /(1+[\text { PretestOdds } \times L R+]) \\
=([7 / 94] \times 16.786) /(1+[[7 / 94\} \times 16.786]) \\
=0.55556=55.6 \%\end{array}$ \\
\hline $\begin{array}{l}\text { Posttest Probability for a negative test } \\
\text { (PosttestProb-) }\end{array}$ & $\begin{array}{l}=(\text { PretestOdds } \times L R-) /(1+(\text { PretestOdds } \times L R-)) \\
=([7 / 94] \times 0.298) /(1+[\{7 / 94\} \times 0.298]) \\
=\mathbf{0 . 0 2 1 7 1}=\mathbf{2 . 1 7 \%}\end{array}$ \\
\hline
\end{tabular}




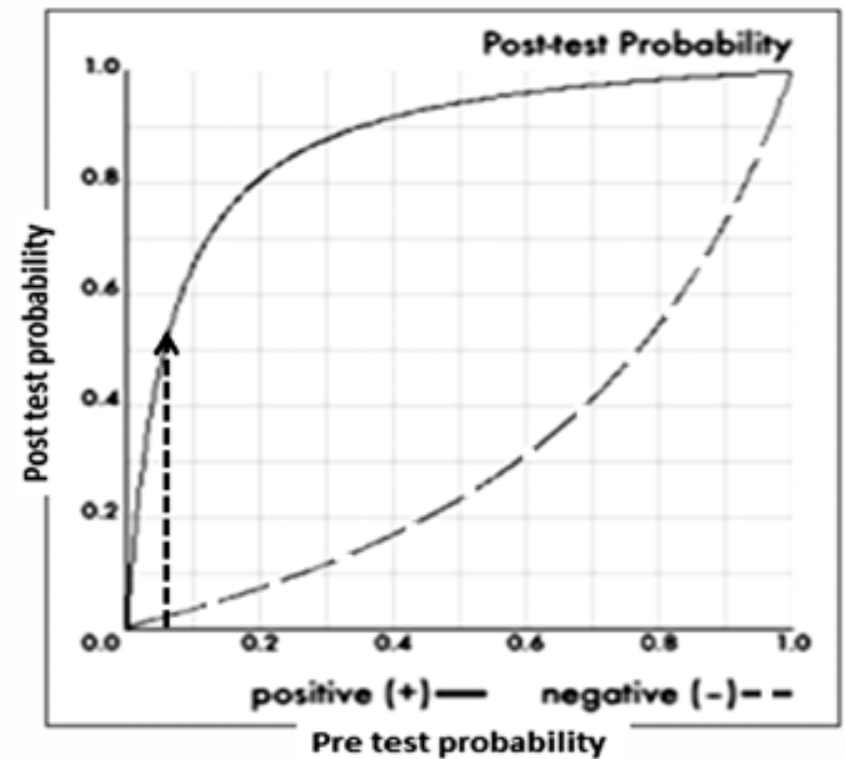

Figure 2. Graph of post-test probabilities for a positive or negative "Baah" test (y-axis) given a pre-test probability for a specific group (x-axis).

In this study population, for example, the prevalence of hearing loss is $6.9 \%$. Prior to the "Baah" test, a child has a $6.9 \%(0.069)$ probability of having hearing loss (tail end of dashed arrow). After conducting the "Baah" test, a specific infant that turns out positive for the test now has $55.6 \%(0.556)$ probability of having hearing loss (head of dashed arrow)."

"Graph created through KT Clearinghouse - Center for Evidence Based Medicine Toronto"

\section{DISCUSSION}

The "Baah" test has a high specificity of $95.7 \%$, which indicates a low false positive rate. Hence, infants that tested positive for "Baah" have a high probability of indeed having a hearing impairment. The rather low sensitivity of $71.4 \%$ (hence a considerable number of false negatives) and positive predictive value of $55.6 \%$ indicate that those that tested negative for "Baah" may still have hearing loss, therefore these infants need to be continually monitored for hearing milestones and parents need to be advised to watch out for any indication of poor speech and/ or language development.

The negative predictive value means that using the "Baah" test, $97.8 \%$ of those without hearing loss were correctly labeled as such.

The likelihood ratio for a positive test means that a positive "Baah" test would be about 17x as likely to be seen in someone with as opposed to someone without hearing loss.

In this study, all infants had a $6.9 \%$ probability of having hearing loss prior to the test (i.e. prevalence). After conducting the "Baah" test, a specific infant that turned out positive for the test now had a $55.6 \%$ probability of having hearing loss (i.e. post-test probability for a positive test). This value may be clinically significant to compel the health care provider to send the infant with a positive "Baah" test for further hearing evaluation. Applying this to the rural setting where OAEs are not readily available, a child that turns out positive for "Baah" test will have a more justifiable reason to be referred to the city for further hearing tests (OAEs, ABR, etc) in order to confirm whether he or she has hearing loss.

For a certain population with a higher prevalence of hearing loss (for example, infants with risk factors such as maternal rubella and use of antibiotics during the neonatal period, etc.), the probability of having hearing loss may be higher after a positive "Baah" test. Figure 2 shows the curve on how the post-test probability would increase depending on the pre-test probability of hearing loss in a specific population. ${ }^{13}$

One limitation of this study is the selection of a gold standard to confirm the diagnosis of hearing loss. At present, there is no single, true gold standard for the diagnosis of newborn hearing loss. Even if an infant passes the OAEs or ABR test, it is still not assured that the child indeed has normal hearing, as $\mathrm{OAE}$ and $\mathrm{ABR}$ are merely physiologic observations that are indirect measures of hearing. Therefore when a child passes the screening test, regardless of the specific tool used, it is advised that the infant be continually monitored for hearing milestones. 1 The most valid confirmatory tool for evaluation of hearing is a behavioral test, which is usually through pure tone audiometry to check whether the individual actually responds to auditory input. 4, 15,16,17 Although these behavioral techniques may be possible for children as young as 6 months old, these techniques are not always easy to perform for this age group, as proper timing and motivation to gain the child's cooperation is necessary to obtain a reliable result. ${ }^{1}$

Despite its limitations, OAEs are still the more popular neonatal hearing screening tool. While OAEs and ABRs are both objective tests, OAE testing is less expensive and easier to administer, while ABR takes a longer time and requires the infant to be asleep. This may necessitate sedation to keep the baby in a quiet state for the duration of the test, although it is often not recommended until after five months of age. OAE results are found to be highly specific (91.8 to $99.7 \%$ specificity) with sensitivity close to $100 \% .{ }^{3} \mathrm{~A}$ local study by Llanes and Chiong also showed that the Otoacoustic Emission test had good concordance with ABR in neonates. ${ }^{18}$ In this study, we selected OAEs as our standard for the presence of newborn hearing loss due to the reasons mentioned above.

This study shows that the reflexive behavioral ("Baah") test has potential as an accurate and cost-effective screening tool to identify infants that may be at greater risk for hearing impairment, and who will need further diagnostic test in a higher level of health care institution. The ability of the test can be assessed in larger population or by field testing in a community-based study. A stronger gold standard for 
measuring the accuracy of the "Baah" test may be used, such as retesting with OAEs or confirmation through $A B R$, as well as possible long-term follow-up to eventually conduct pure tone audiometry when the child is cooperative enough for a behavioral test.

The use of this test may lead to earlier detection of hearing impairment for infants in the rural communities who cannot be readily tested with $O A E s$ or $A B R$. This may provide greater awareness to the community and lead to more referrals with more sophisticated screening and diagnostic tools.

Should this test be considered in community practice, the production of the sound stimulus "Baah" may be taught to community health workers during their training seminars, to be able to produce a standardized stimulus, as well as be trained to assess an infant's normal reactions to sound. It may also be worthwhile to investigate the utility of "Baah" test in combination with the evaluation of risk factors and hearing milestones of infants, which might result in a more sensitive community-based newborn hearing screening program.

\section{ACKNOWLEDGEMENTS}

We thank Dr. Rina Reyes-Quintos, for her review of the initial proposal; Ms. Aneezah Joy Mutuc and Ms. Floriza Araneta, our participating investigators, and Dr. Patrick Labra for his assistance with the newborns. We also thank those who helped with the PSO-HNS Analytical Research Contest: Dr. Romeo Villarta for his ideas and inputs on the presentation, Dr. Warren Holgado and Dr. Rikka Ramos for the paper submission, and Dr. Marion Acuin and Dr. Micaela Pacis-Vinarao for covering for the other hospital duties.

\section{REFERENCES}

1. Cummings CW, Flint PW, Haughey BH, Robbins KT, Thomas JR, Harker LA, et al, editors. Cummings Otolaryngology-Head \&Neck Surgery.4th ed. Philadelphia: Mosby, Inc; 2005. p. 4387-4390.

2. Wrightson AS. Universal newborn hearing screening. Am Fam Physician. 2007 May; 75(9): 13491352.

3. Freitas VS, Alvarenga KF, Bevilacqua MC, Martinez MAN, Costa OA. Critical analysis of three newborn hearing screening protocols (original title: Análise crítica de três protocolos de triagem auditiva neonatal). Pró-Fono Revista de Atualização Científica. 2009 Jul-Sep; 21(3):201-6.

4. Porter HL, Neely ST, Gorga MP. Using benefit-cost ratio to select universal newborn hearing screening test criteria. Ear Hear. 2009 Aug; 30(4): 447-457.

5. Republic Act 9709. An act establishing a universal newborn hearing screening program for the prevention, early diagnosis and intervention of hearing loss. Republic of the Philippines, Fourteenth Congress, Second Regular Session, Metro Manila. Senate and House of Representatives of the Philippines. 28 Jul 2008.

6. Olusanya BO. Addressing the global neglect of childhood hearing impairment in developing countries. PLoS Med. 2007 Apr; 4(4):e74. Erratum in: PLoS Med. 2007 Jun; 4(6):e203. [cited January 2010]. Available from: www.plosmedicine.org.

7. Santos-Cortez R, Chiong CM. Cost-effectiveness of universal hearing screening in the Philippines. Acta Medica Philippina. Submitted 2011.

8. UNICEF. At a glance: Philippines. [Cited September 2011]. Available from: http://www.unicef.org/ infobycountry/philippines_statistics.html

9. United Nations, Department of Economic and Social Affairs, Population Division: World population prospects. New York. [Updated 2010, cited September 2011]. Available from: http:// esa.un.org/unpd/wpp/country-profiles/pdf/608.pdf

10. Mencher GT, Davis AC, DeVoe SJ, Beresford D, Bamford JM. Universal neonatal hearing screening: past, present, and future. Am J Audiol. 2001 Jun; 10(1):3-12.

11. McCormick, B. Neonatal hearing screening and assessment: the distraction test as a procedure for hearing screening - a recommended test protocol. [Updated August 13, 2002, cited January 2010]. Available from: www.nhsp.info/prots.shtml.

12. Abes FLL, Gloria-Cruz TL, Abes GT. The voice test as an alternative hearing test for the universal newborn hearing screening program in the Philippines. Acta Medica Philippina. Submitted 2011.

13. KT Clearinghouse - Center for Evidence Based Medicine Toronto - Statistical Calculator. Toronto Canada. (c) 2000-2012. [Cited September 2011]. Available from: http://ktclearinghouse.ca/ cebm/practise/ca/calculators/statscalc

14. GraphPad Software. QuickCalcs, Online Calculators for Scientists. GraphPad Software, Inc. CA USA. ๑ 2002-2005, [Cited April 2012]. Available from: http://www.graphpad.com/quickcalcs/ contingency1.cfm

15. Kennedy C, McCann D. Universal neonatal hearing screening moving from evidence to practice. Arch Dis Child Fetal Neonatal Ed. 2004 Sep; 89(5):F378-F383.

16. Declau F, Boudewyns A, Van den Ende J, Peeters A, van den Heyning P. Etiologic and audiologic evaluations after universal neonatal hearing screening: analysis of 170 referred neonates. Pediatrics. 2008 Jun; 121(6): 1119-26.

17. American Speech-Language-Hearing Association. (2004). Guidelines for the Audiologic Assessment of Children From Birth to 5 Years of Age [Guidelines]. Available from www.asha. org/policy.

18. Llanes EGD, Chiong CM: Evoked otoacoustic emissions and auditory brainstem responses: Concordance in hearing screening among high risk children. Acta Otolaryngol 2004; 124:387390. 